\title{
Phase Synchronization Based on Regions Heterogeneity in Suprachiasmatic Nucleus
}

\author{
Qingduan Fan ${ }^{1,2, a}$, Zengrong Liu $^{1, b}$ \\ ${ }^{1}$ Institute of Systems Biology, Shanghai University, Shanghai, 200444, China \\ ${ }^{2}$ College of Fundamental Studies, Shanghai University of Engineering Science, Shanghai, 201620, \\ China \\ aemail:fanqingduan@163.com, bemail:zrongliu@126.com, ${ }^{*}$ corresponding author
}

\begin{abstract}
Keywords: SCN network, Kuramoto model, phase synchronization/entrainment
\end{abstract}
\begin{abstract}
Based on the suprachiasmatic nucleus (SCN) structure and molecular biology, numerous significant results have been obtained via some well-designed network-models as regards to circadian rhythm, in which synchronized cells can be entrained by a 24-h light-dark cycle. However, existing models cannot explain the phase synchronization clearly, such as jet lag. In this paper, a modified Kuramoto model in SCN network is proposed. Results show that the model exhibits some prominent characteristics of phase synchronization. The results obtained in this model also provide some deep insights for the mechanism of circadian rhythm, and lay a foundation for further discussion and analysis.
\end{abstract}

\section{Introduction}

Mammalian behavior rhythms are dominated by a central pacemaker located in the suprachiasmatic nucleus (SCN), which is composed of about 20,000 oscillating neurons [1]. The dissociated neurons have scattered periods between 20 and 28 hours [2]. There are quantities of research on circadian rhythm in single cell level $[3,4]$. According to these research, several clock proteins exhibit periodical oscillation, including PERIOD(PER1,2,3), FRQENCY(FRQ1,2), CRYPTOCHROME(CRY1,2). Hence, it is reasonable to assume that limit cycles can be generated in the circadian system [5]. Based on these facts, Goodwin model, Poincare model and some Kuramoto-type models have been applied to circadian system [6-9].

SCN can be divided into ventral-lateral (VL) subregion and dorsal-medial (DM) subregion, which have different functions and topologies. VL and DM are light sensitive and insensitive respectively. VL, about a quarter of SCN, is entrained by the external light-dark cycle, while DM shows free-running oscillation except that it receives light signals via VL [10]. In topology, VL is a small-world network while DM is a nearest neighbor network, moreover, neurons in VL directly affect neurons in DM and the intercellular coupling in DM is weak [10].

Accordingly, it is necessary to divide SCN into VL and DM to discuss phases of neuronal oscillators. Neurons in SCN form a network consists of heterogeneous oscillators, which emerges a robust circadian rhythm. Although the structures of CRY, CLOCK and BMAL have been verified, the mechanism of circadian rhythms is still not clear.

To explain the mechanism of circadian rhythms, phase synchronization is also necessary to be considered as well as period synchronization. In this paper, a modified Kuramoto model is proposed to discuss the effect of structure parameter on the phase synchronization.

\section{The modified Kuramoto model}

Neural oscillators have the similar periods and phases to make the circadian rhythm work well. So it is necessary to discuss phase synchronization or entrainment. In recent research [11], VL is denoted with a WS small-world network matrix A, while DM is denoted with a cyclic matrix B. Based on single-direction dissemination of light signal, the coupling from VL to DM is denoted by 
C. Connectivity matrix of neurons in SCN can be described as $D=\left(\begin{array}{cc}A & C \\ 0 & B\end{array}\right)$, where $D_{i j}$ indicates a directed connectivity from neuron i to neuron $\mathrm{j}$.

For simplicity, periodical light signal is denoted by sinusoidal function, and the modified Kuramoto model system is displayed as follows.

$$
\dot{\theta}_{i}= \begin{cases}\omega_{i}+\frac{1}{v(i)} \sum_{j=1}^{N_{1}} s c_{1} D_{i j} \sin \left(\theta_{j}-\theta_{i}\right)+a \sin \left(\Omega t-\theta_{i}\right) & 1 \leq i \leq N \\ \omega_{i}+\frac{1}{v(i)}\left(\sum_{j=1}^{N_{1}} s c_{1} D_{i j} \sin \left(\theta_{j}-\theta_{i}\right)+\sum_{j=N_{1}+1}^{N} s c_{2} D_{i j} \sin \left(\theta_{j}-\theta_{i}\right)\right) & \end{cases}
$$

The strength of the couplings (sc) in VL and DM are constants. Here the strength of the coupling $\mathrm{Sc}_{2}$ in $\mathrm{DM}$ and from VL to DM is one-tenth of $\mathrm{Sc}_{1}$ in VL.

Table.1 Parameters in system (1)

\begin{tabular}{c|l|l}
\hline $\begin{array}{c}\text { Parameter } \\
\text { symbol }\end{array}$ & Meaning of parameter & Value \\
\hline$N$ & Number of neurons in SCN & 2000 \\
\hline$N_{1} / N_{2}$ & Number of neurons in VL and DM & $500 / 1500$ \\
\hline$w_{i}$ & Natural frequency of oscillators in VL & $1 / N(24,1)$ \\
\hline $\mathrm{K}$ & Number of neighbors on each side of neurons & 3 \\
\hline $\mathrm{p}$ & Reconnecting probability of nodes in VL & 0.1 \\
\hline $\mathrm{q}$ & Connecting probability of nodes from VL to DM & 0.006 \\
\hline $\mathrm{SC} 1$ & Strength of coupling between neurons in VL and from VL to DM & 0.1 \\
\hline $\mathrm{SC} 2$ & Strength of coupling between neurons in DM & 0.01 \\
\hline$\alpha$ & Strength of coupling from light signal to neurons in VL & 0.1 \\
\hline$v(i)$ & The number of neurons coupling neuron $i$ & \\
\hline & &
\end{tabular}

\section{Results}

Under a periodic light signal, phases of neuronal oscillators are entrained to the external signal. Parameters are displayed in table 1, and the initial values of VL and DM are respectively uniformly distributed on $[0,2 \pi]$, and the time step is 1 hour. The simulations of systems (1) are shown in Fig.1. It implies that neuronal oscillators with different phases are entrained to 24-h cycle light signal. Most neurons in DM synchronize with the light signal. The rest sustains free-running oscillation, which may be caused by sparse and weak coupling.

In a circadian rhythm, phases should have a high-quality synchronization so as to transfer the rhythm to peripheral organization. $\theta_{\mathrm{j}}$ means the phase of neuronal oscillator j. Based on ref.[14], the order parameters of phases in VL, DM and SCN are denoted respectively by $\mathrm{r}_{1}, \mathrm{r}_{2}$ and r. i.e.

$$
r_{1}(t)=\left|\frac{1}{N_{1}} \sum_{j=1}^{N_{1}} e^{i \theta_{j}(t)}\right|, r_{2}(t)=\left|\frac{1}{N_{2}} \sum_{j=1}^{N_{2}} e^{i \theta_{j}(t)}\right| \text { and } r(t)=\left|\frac{1}{N} \sum_{j=1}^{N} e^{i \theta_{j}(t)}\right|
$$

The structure and dynamic of the network are the key factors to the synchronization quality. When $\mathrm{K}$ is assigned to $1,2,3,4,5$ and 6, the order parameters in VL, DM and SCN during 120 hours are shown in Fig. 2. It is shown that $r_{1}$ stays close to 1 for a short time while $r_{2}$ and $r$ reach a stable level. That is, neurons in VL rapidly synchronize to the light signal but synchronization among neurons in DM has a ceiling. Interestingly, as $\mathrm{K}$ increases, $r_{1}$ increases while $r_{2}$ and $r$ decrease. The phenomena may result from the small mean degree and heterogeneous frequencies of SCN network. In other words, high complexity results in neurons in SCN being difficult to entrain. When $\mathrm{K}$ is $3, \mathrm{r}$ is 0.63 [6]. In later simulation, $\mathrm{K}$ is taken as 3 and time interval is 120 hours. 


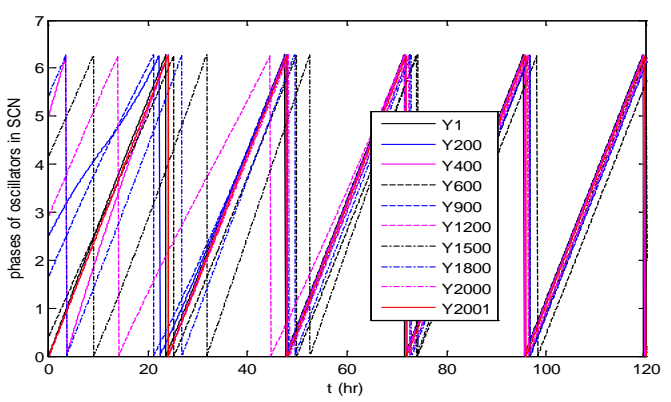

Fig.1. Phases synchronize to the 24h-cycle light signal. Phases in VL and DM are denoted respectively by solid and dotted lines.
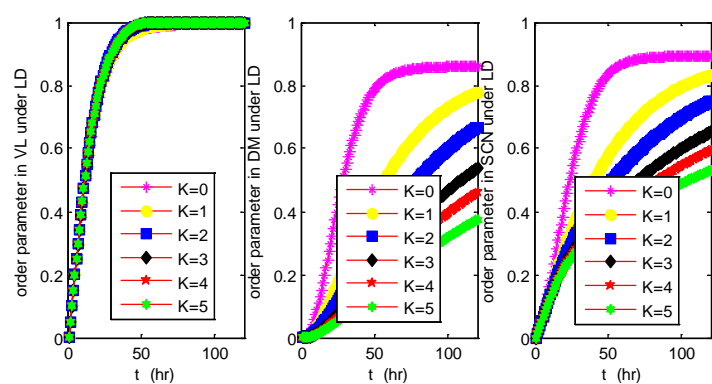

Fig.2. Effect of degree on order parameters in VL, DM and SCN. Order parameters of output rhythm decrease as K increases.

Phase dynamic can be affected by both light intensity and coupling strength. $a$ and sc are taken respectively from 0 to 0.1 with step size 0.02 , and other parameters and initial values are the same as that in Fig.1. Simulations are shown respectively in Fig.3 and Fig.4. The greater light intensity is, the greater order parameters are. This result is consistent with the practicality that a person feels sleepy on overcast days while refreshed on sunny days. Fig. 4 indicates that order parameters $r_{2}$ and $\mathrm{r}$ increase as sc increases, while $r_{1}$ is close to 1 due to the direct induction by light signal.
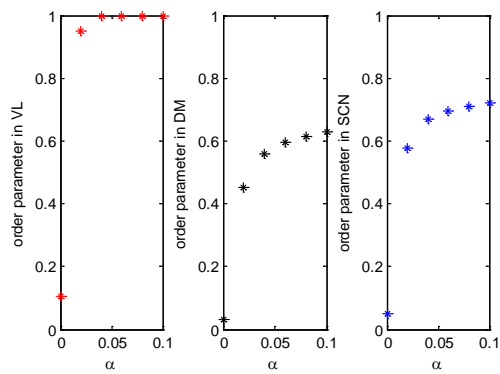

Fig.3. Effect of light density on order parameters of neuron oscillators in VL, DM and SCN. All order parameters increase with the increase of light density.
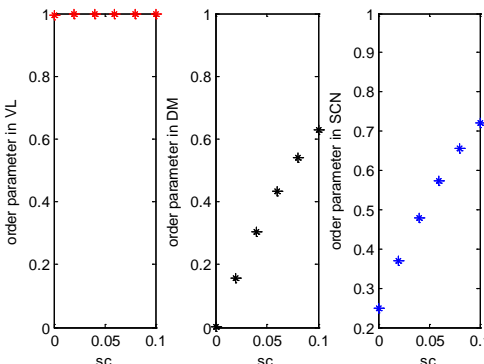

Fig.4. Effect of parameter sc on order parameters of neuron oscillators in VL, DM and SCN. All order parameters increase with the increase of coupling.

\section{Conclusion}

In this paper, a modified Kuramoto model is proposed based on region heterogeneity in SCN network. And some prominent characters of phase synchronization to sunlight are obtained. Simulations show that the entrainment ability correlates positively with the light intensity and coupling strength, while it correlates negatively with the mean degree, which may be attributed to sparse coupling between neurons and heterogeneous frequencies of neurons.

\section{Acknowledgment}

We acknowledge the support grants from National Natural Science Foundation of China (Grant No. 10832006, 11172158 and 11101265).

\section{References}

[1] Hastings MH, Herzog ED: Clock genes, oscillators, and cellular networks in the suprachiasmatic nuclei. Journal of biological rhythms 2004, 19(5):400-413.

[2] Honma S, Nakamura W, Shirakawa T, Honma K: Diversity in the circadian periods of single neurons of the rat suprachiasmatic nucleus depends on nuclear structure and intrinsic period. Neuroscience letters 2004, 358(3):173-176.

[3] Leloup JC, Goldbeter A: Toward a detailed computational model for the mammalian circadian clock. Proceedings of the National Academy of Sciences of the United States of America 2003, 100(12):7051-7056. 
[4] Goodwin BC: Oscillatory behavior in enzymatic control processes. Advances in enzyme regulation.1965, 3:425-438.

[5] Kuramoto Y: Chemical oscillations, waves, and turbulence, Dover edn. Mineola, N.Y.: Dover Publications; 2003.

[6] Gonze D, Bernard S, Waltermann C, Kramer A, Herzel H: Spontaneous synchronization of coupled circadian oscillators. Biophysical journal 2005, 89(1):120-129.

[7] Ramkisoensing A, Gu C, van Engeldorp Gastelaars HM, Michel S, Deboer T, Rohling JH, Meijer JH: Enhanced phase resetting in the synchronized suprachiasmatic nucleus network. Journal of biological rhythms 2014, 29(1):4-15.

[8] Ananthasubramaniam B, Herzog ED, Herzel H: Timing of neuropeptide coupling determines synchrony and entrainment in the mammalian circadian clock. PLoS computational biology 2014, 10(4):e1003565.

[9] Li Y, Liu ZR: Synchronization of Clocks Coupled by Neurotransmitter in the Scn. J Biol Syst 2013, 21(1).

[10] Yamaguchi S, Isejima H, Matsuo T, Okura R, Yagita K, Kobayashi M, Okamura H: Synchronization of cellular clocks in the suprachiasmatic nucleus. Science 2003, 302(5649):1408-1412.

[11] Vasalou C, Henson MA: A multicellular model for differential regulation of circadian signals in the core and shell regions of the suprachiasmatic nucleus. Journal of theoretical biology 2011, 288:44-56.

[12] Arenas A, Diaz-Guilera A: Synchronization and modularity in complex networks. Eur Phys J-Spec Top 2007, 143:19-25. 